\title{
Assessment of oxygen, oxyanions and trace metals benthic fluxes by a novel non-invasive technique in coastal areas.
}

\author{
JULIE REGIS ${ }^{1}$, SAMUEL MEULÉ ${ }^{2}$, PATRICK VERDOUX ${ }^{3}$, \\ ISABELLE TECHER ${ }^{3}$ AND SYLVAIN RIGAUD ${ }^{3}$ \\ ${ }^{1}$ Univ. Nîmes, EA 7352 CHROME \\ ${ }^{2}$ Aix Marseille Univ, CNRS, IRD, INRAE, Coll France, \\ CEREGE, Aix-en-Provence, France \\ ${ }^{3}$ Univ. Nîmes, EA 7352 CHROME, rue du Dr Georges Salan, \\ 30021 Nîmes, France
}

Presenting Author: julie.regis@unimes.fr

Because of anthropogenic forcing and global warming, Mediterranean lagoons are increasingly impacted by deoxygenation events that mostly occur over short (i.e., hours and days) timescales. Oxygen is the most important driver of biogeochemical processes occurring in surface sediment and its temporal variation is known to impact the chemical transfer of pollutants (e.g., excess nutrients, metals, metalloids), at the sediment-water interface. However, a lack of knowledge remains on the impact of short-term oxygen variations on the benthic fluxes. A fully equipped structure called BEATRISS (for Benthic Exchange Autonomous Time-series Recording In Situ System) has been set up to record benthic fluxes at those short-time scales. The new technique relies on the coupling of vertical chemical concentration gradients and turbulent diffusion coefficient in the benthic boundary layer. The structure was for the first time deployed during a two-weeks period in the Berre and Thau lagoons, two Mediterranean lagoons known to be affected by deoxygenation events. Continuous oxygen gradients were obtained using five autonomous probes while oxyanions and trace metals integrated gradients were measured using passive samplers (i.e., DGT). Turbulent diffusion coefficient was determined from high resolution current velocity measured with two current meters. In addition, environmental conditions (salinity, light, temperature, turbidity and pressure) were monitored by specific probes. The calculation of the oxygen fluxes was verified with a commonly accepted approach (i.e., sediment core incubation) for each site. First results showed an intense and rapid dynamic between the oxygen consumption and production of the sediments at the diurnal cycle. Local currents, mainly controlled by tides, wind forcing, also seem to have an impact on this dynamic. Oxyanions and trace metals also exhibited significant vertical gradients in the benthic layer and time-integrated flux were obtained and discussed with respect of oxygen variations for each lagoon. 